\title{
Evaluation of Thyroid Dysfunction in Patients with Head and Neck Cancer Receiving Radiation Therapy or Chemoradiation
}

\author{
Vivek Immanuel ${ }^{1}$, Jaineet Sachdeva², Navjot Singh ${ }^{3}$, Pamela Alice Kingsley ${ }^{4}$, Rajesh Chandran ${ }^{5}$ \\ ${ }^{1}$ Senior Resident, Department of Radiation Oncology, Christian Medical College \& Hospital, Ludhiana, Punjab, India. \\ ${ }^{2}$ Professor \& HOD, Department of Radiation Oncology, Christian Medical College \& Hospital, Ludhiana, Punjab, India. \\ ${ }^{3}$ Professor \& HOD, Department of General Medicine, Christian Medical College \& Hospital, Ludhiana, Punjab, India. \\ ${ }^{4}$ Professor, Department of Radiation Oncology, Christian Medical College \& Hospital, Ludhiana, Punjab, India. \\ ${ }^{5}$ Medical Physicist, Department of Radiation Oncology, Christian Medical College \& Hospital, Ludhiana, Punjab, India.
}

\section{ABSTRACT}

\section{BACKGROUND}

Majority of head and neck tumours are locoregionally advanced at the time of diagnosis. Concurrent chemoradiotherapy is the standard of care for these patients. Radiation portals cover the primary tumour along with the whole neck, thereby including the thyroid gland in the radiation field leading to its dysfunction. The incidence of thyroid dysfunction varies from $10-46 \%$ and manifests as elevated TSH levels. We wanted to identify the incidence of thyroid dysfunction in head and neck cancer patients receiving radiation therapy or chemoradiotherapy and explore the association between radiation dose to the thyroid and thyroid dysfunction.

\section{METHODS}

This one-year prospective study was conducted in 82 patients with histopathologically proven head and neck cancer receiving 3D conformal radiotherapy to the neck from a 6MV linear accelerator. Patients with history of previous thyroid surgery, pre-existing thyroid disease and recurrent disease were excluded. Free T3, free T4, and TSH were obtained at baseline and then at 3 monthly intervals up to 12 months after completion of treatment.

\section{RESULTS}

In our study, the incidence of thyroid dysfunction was found to be $19.51 \%$. All the cases of thyroid dysfunction had subclinical hypothyroidism. Higher mean dose to the thyroid and smaller volume of thyroid gland were found to be associated with hypothyroidism. There was no association between thyroid dysfunction and dosevolume parameters V10-V50 and primary site of involvement. Older patients were more likely to develop hypothyroidism. No association was seen between gender and mode of treatment and thyroid dysfunction.

\section{CONCLUSIONS}

Thyroid dysfunction after head and neck cancer continues to be underestimated and under reported, as symptoms of thyroid dysfunction may go unnoticed under the cover of other associated comorbidities and side effects of the treatment modalities. Regular testing of thyroid function in patients undergoing radiation or chemo radiation can help in diagnosing thyroid dysfunction at the subclinical stage. Larger multi-institutional studies with longer follow-up are needed to further study the morbidity associated with post radiation thyroid dysfunction.

\section{KEY WORDS}

Head and Neck Cancer, Chemoradiation, Thyroid Dysfunction
Corresponding Author:

Vivek Immanuel,

\#224-A Model Town Extension,

Ludhiana-141002, Punjab, India.

E-mail:vivekimmanuel1313@yahoo.co.in

DOI: 10.14260/jemds/2019/684

Financial or Other Competing Interests: None.

How to Cite This Article:

Immanuel V, Sachdeva J, Singh $N$, et al. Evaluation of thyroid dysfunction in patients with head and neck cancer receiving radiation therapy or chemoradiation. J. Evolution Med. Dent. Sci. 2019;8(42):3155-3160, DOI: $10.14260 / \mathrm{jemds} / 2019 / 684$

Submission 15-03-2019,

Peer Review 01-10-2019,

Acceptance 08-10-2019,

Published 21-10-2019. 


\section{BACKGROUND}

Head and neck cancers refer to neoplasms arising from the skull base to the region of the thoracic inlet. Approximately 50,000 patients are diagnosed annually with squamous cell carcinoma head and neck in the United States. Worldwide, approximately 600,000 patients are afflicted. ${ }^{1}$ Around 57.5\% of global head and neck cancers occur in Asia. ${ }^{2}$ Around 60\% of these patients present with locally advanced non metastatic disease. ${ }^{3}$

Radiotherapy was the most commonly used non-surgical therapy for locally advanced disease. But Radiotherapy with concurrent chemotherapy has been found to be superior to radiotherapy alone in the management of locally advanced head and neck cancers. This is because chemotherapeutic agents may both sensitize the cells to radiation and may also produce additional cytotoxicity. Data from the MACH-NC trial demonstrated that the use of radiotherapy with concurrent chemotherapy resulted in reduction in risk of death and improvement in 5-year survival in patients of head and neck cancer. ${ }^{4}$

However, patients who receive radiotherapy to the neck are at increased risk of developing thyroid dysfunction, which is usually seen in the form of biochemical hypothyroidism but may also present as clinical hypothyroidism or thyrotoxicosis. These patients are also more likely to develop both benign and malignant nodules of the thyroid gland. 5 Thyroid dysfunction after radiation is most often manifested by elevated serum concentration of Thyroid stimulating hormone (TSH).

The incidence of hypothyroidism and the time after which it develops following radiation, tends to vary. According to Akgun et al $^{6} 10 \%$ patients developed hypothyroidism within 6 months of radiotherapy and 23\% developed hypothyroidism within 12 months of completion of radiotherapy. At a median follow up of 47 months, 52\% patients were found to be hypothyroid. According to Kim et al, $746 \%$ patients developed hypothyroidism at a median period of 8 months after completion of radiotherapy. Weissler and Berry ${ }^{8}$ reported that $57 \%$ of patients developed elevated TSH levels after radiotherapy. Banipal et al $^{9}$ found TSH elevation in $75.4 \%$ patients who received radiation for the treatment of head and neck cancers.

Radiation dose may also influence the incidence of hypothyroidism. According to Bhandare et al, ${ }^{10}$ significantly higher incidence of hypothyroidism was found in patients receiving a mean thyroid gland dose more than 45 Gy. Sachdev et al ${ }^{11}$ found that V50 $>60 \%$ puts patients at a significantly higher risk of becoming hypothyroid.

Thyroid volume is a significant prognostic factor in the development of radiation induced hypothyroidism, with an increased risk at lower thyroid volumes. Jerezeck et $\mathrm{al}^{12}$ showed that smaller thyroid volume was associated with higher incidence of thyroid toxicity. In 2012, Boomsma et al ${ }^{13}$ reported that the probability of hypothyroidism increased with increase in mean dose and decreased with higher thyroid gland volume.

Thyroid disorders after radiation therapy to the neck still represent a clinically underestimated problem.

\section{METHODS}

It was a one-year prospective study carried out from $1^{\text {st }}$ November 2015 to $31^{\text {st }}$ October 2016 and comprised of 82 patients with a histopathological diagnosis of squamous cell carcinoma head and neck, planned for therapeutic radiation therapy or chemoradiation. Patients with history of, thyroid dysfunction, prior exposure to radiation therapy or chemoradiation or of thyroid surgery were excluded from the study. Informed consent was taken from eligible patients for participation in the study. All eligible patients underwent a complete clinical history and physical examination. Blood investigations including complete blood count, renal function test and thyroid function test along with relevant radiological investigations and staging was done. All patients were staged according to criteria established by the American Joint Committee on Cancer.

EBRT was delivered by Elekta Compact 6MV linear accelerator by 3-Dimensional conformal radiotherapy (3DCRT). Patients planned for 3DCRT underwent a contrast enhanced CT simulation using $3 \mathrm{~mm}$ slice thickness in supine position with head and neck rest, or fit cast and shoulder traction. The thyroid gland was contoured to calculate the dose to the thyroid. The plans were generated using the Treatment planning system CMS XiO 4.6. A dose of 40 Gy in 20 fractions was delivered in 4 weeks followed by a boost of 20-30 Gy in 10-15 fractions over 2-3 weeks.

Patients planned for chemoradiation were given Inj. Cisplatin $\left(40 \mathrm{mg} / \mathrm{m}^{2}\right)$ on a weekly or $\left(100 \mathrm{mg} / \mathrm{m}^{2}\right)$ three weekly basis or Inj. Carboplatin (AUC-2) on a weekly basis. Thyroid function test (TFT) was done at baseline and periodically at 3-month intervals after completion of radiation or chemoradiation for a minimum period of 6 months. Radiation induced thyroid dysfunction was determined with an endpoint of abnormal thyroid stimulating hormone (TSH), free Triiodothyronine (FT3) and thyroxine (FT4) levels. A diagnosis of hypothyroidism was based on TSH value greater than the upper limit of the laboratory range. The normal range of thyroid function parameters is- 1 . triiodothyronine (fT3)- 3.1-6.8 pmol/L, 2. tetraiodothyronine (fT4)- 12-22 pmol/L, 3. thyroid stimulating hormone (TSH)$02-4.2 \mu \mathrm{U} / \mathrm{ml}$ The radiation dose received by the thyroid was calculated as mean dose, maximum dose and dose- volume parameters V10-V50.

\section{Statistical Analysis}

Categorical variables were presented in number and percentage (\%) and continuous variables were presented as mean \pm SD and median. Normality of data was tested by Kolmogorov-Smirnov test. If the normality was rejected, then nonparametric test was used. Statistical tests were applied as follows-1. Quantitative variables were compared using Independent T test/Mann-Whitney Test (When the data sets were not normally distributed) between the two groups and Paired T test/ Wilcoxon test for pair wise comparison across follow up and repeated measure ANOVA for comparison across follow up. 2. Qualitative variables were correlated using Chi-Square test/Fisher exact test. 3. Pearson correlation coefficient/Spearman rank correlation coefficient was used to 
assess the association of various parameters with each other. A p value of $<0.05$ was considered statistically significant. The data was entered in MS EXCEL spreadsheet and analysis was done using Statistical Package for Social Sciences (SPSS) version 21.0

\section{RESULTS}

The study included 82 patients of Head and neck cancer treated with 3DCRT.

\begin{tabular}{|c|c|c|}
\hline Age & Frequency & Percentage (\%) \\
\hline $26-35$ & 12 & 14.63 \\
\hline $36-45$ & 10 & 12.20 \\
\hline $46-55$ & 19 & 23.17 \\
\hline $56-65$ & 25 & 30.49 \\
\hline $66-75$ & 11 & 13.41 \\
\hline$>75$ & 5 & 6.10 \\
\hline \multicolumn{3}{|l|}{ Sex } \\
\hline Males & 76 & 93 \\
\hline Females & 6 & 7 \\
\hline \multicolumn{3}{|l|}{ Primary Site } \\
\hline Oral cavity & 39 & 47.56 \\
\hline Larynx & 19 & 23.17 \\
\hline Oropharynx & 13 & 15.85 \\
\hline Maxilla & 5 & 6.19 \\
\hline Hypopharynx & 5 & 6.10 \\
\hline Nasopharynx & 1 & 1.22 \\
\hline \multicolumn{3}{|l|}{ Mode of Treatment } \\
\hline Chemoradiation & 70 & 85.37 \\
\hline Radiation Alone & 12 & 14.63 \\
\hline \multicolumn{3}{|l|}{ Chemo-Regimen } \\
\hline Weekly CDDP & 46 & 66 \\
\hline 3 weekly CDDP & 15 & 21 \\
\hline Weekly Carboplatin & 9 & 13 \\
\hline \multicolumn{3}{|l|}{ Radiation Dose } \\
\hline$<60 \mathrm{~Gy}$ & 6 & 7.23 \\
\hline 60 Gy & 23 & 28.05 \\
\hline$>60 \mathrm{~Gy}$ & 53 & 64.63 \\
\hline \multicolumn{3}{|l|}{ Follow-Up } \\
\hline 6 Months & 67 & 81.71 \\
\hline 9 Months & 11 & 13.41 \\
\hline 12 Months & 4 & 4.88 \\
\hline
\end{tabular}

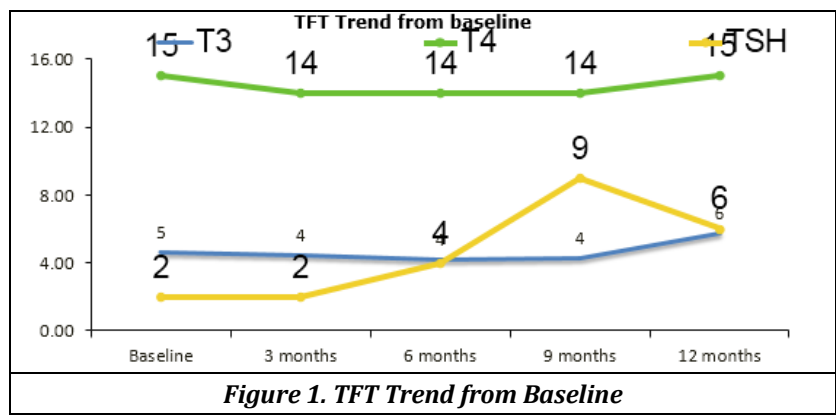

There was a statistically significant decrease in free T3 levels at 6 months post completion of treatment(p value $<0.05)$.The variation in T3 levels showed no statistically significant change when compared to age group, mode of treatment or radiation dose. There was a statistically significant decrease in free $\mathrm{T} 4$ levels from baseline at 3 months and 6 months ( $p$ value $<0.05$ ). Beyond 6 months of follow-up, there was an increase in the mean free T4 levels at 9 months and 12 months, which was not statistically significant. There was no statistically significant change in T4 levels when it was assessed according to separate age groups, mode of treatment or total radiation dose.

The incidence of hypothyroidism was $19.51 \%$. The incidence was $2.43 \%$ at 3 months and increased steadily till it was $19.51 \%$ at 9 months onwards. The increase in TSH levels from baseline, at 3 months and 6 months was found to be statistically significant. There was no positive correlation between TSH levels and age group or mode of treatment.

The increase in TSH from baseline in the patients receiving $<60$ Gy was found to be statistically significant ( $p$ value $<005$ ). The increase in TSH levels was not found to be statistically significant in patients receiving 60Gy and above. In the patients receiving $<60 \mathrm{~Gy}-33.33 \%, 60 \mathrm{~Gy}-4.35 \%$ and $>60$ Gy- 24.52\% developed hypothyroidism. These differences were found to be statistically significant ( $p$ value $<0.05$ ). Majority of the hypothyroid patients were more than 56 years of age. In the $>75$-year age group, it was seen that $80 \%$ of patients developed hypothyroidism.

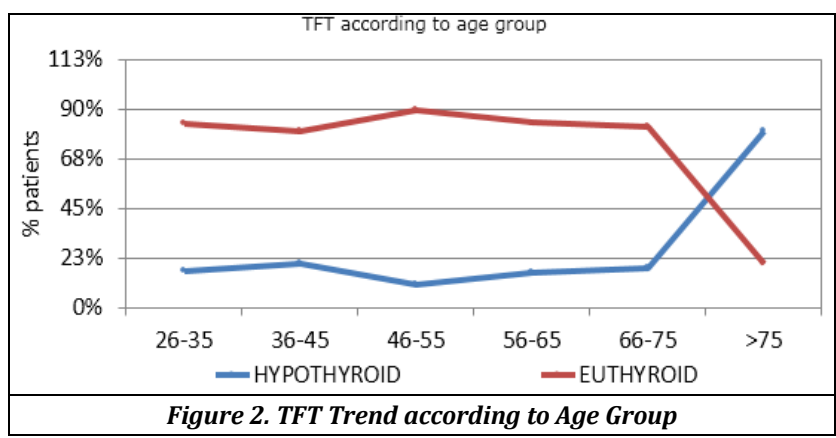

The mean maximum dose was higher in the hypothyroid group compared to the euthyroid group, however, not found to be statistically significant.

\begin{tabular}{|c|c|c|c|}
\hline & \multicolumn{2}{|c|}{ TSH } & \multirow[b]{2}{*}{$\mathbf{p}$} \\
\hline & Hypothyroid $n=16$ & Euthyroid n=66 & \\
\hline \multicolumn{3}{|l|}{ Max Dose } & \multirow{5}{*}{0.445} \\
\hline Mean \pm SD & $5273.5 \pm 66.92$ & $5227.52 \pm 236.31$ & \\
\hline Median & 5258 & 5257 & \\
\hline Min-Max & $5175-5434$ & $3879-5437$ & \\
\hline Inter quartile Range & $5225.500-5335.500$ & $5222-5313$ & \\
\hline \multicolumn{3}{|l|}{ Mean Dose } & \multirow{5}{*}{0.001} \\
\hline Mean \pm SD & $5004.69 \pm 161.91$ & $4643.44 \pm 825.05$ & \\
\hline Median & 5029.5 & 4878 & \\
\hline Min-Max & $4621-5297$ & $1105-5136$ & \\
\hline Inter quartile Range & $4912.500-5108.500$ & $4675-5065$ & \\
\hline \multicolumn{4}{|c|}{ Table 2. Thyroid Dose Parameters } \\
\hline
\end{tabular}

Hypothyroid patients were found to have a higher mean dose compared to the euthyroid patients. This difference was found to be statistically significant ( $p$ value $<0.05$ ). The mean volume of the thyroid was higher in the euthyroid group compared to the hypothyroid group and this difference was found to be statistically significant ( $p$ value $<0.05$ ).

\begin{tabular}{|c|c|c|c|}
\hline Thyroid Volume & Hypothyroid & Euthyroid & p \\
\hline Sample size & 16 & 66 & \multirow{2}{*}{0.026} \\
\hline Mean \pm Stdev & $9.92 \pm 2.89$ & $12.75 \pm 4.78$ & \\
\hline Median & 10.2 & 11.74 & \\
\hline Min-Max & $4.21-15.22$ & $3.24-29.62$ & \\
\cline { 1 - 2 } Inter quartile Range & $8.820-12.220$ & $10.200-13.660$ & \\
\cline { 1 - 1 } & Table 3. Thyroid Volume \\
\hline
\end{tabular}

When thyroid function was compared to dose volume parameters V10, V20, V30, V40 and V50, it was seen that V10, V20, V30 was found to be higher in the hypothyroid group, V40 was found to be higher in the euthyroid group and V50 was found to be higher in the hypothyroid group. However, these differences were not found to be statistically significant. 


\section{DISCUSSION}

The thyroid gland was previously considered to be relatively radio resistant. However, it was seen that patients exposed to radiation such as those receiving radiation to the head and neck for treatment of cancers, those exposed to radiation as a result of nuclear accidents and the nuclear explosions in Hiroshima and Nagasaki, showed an increased incidence of thyroid dysfunction and thyroid cancers.

Radiation is thought to act on thyroid cells by direct damage to the parenchymal cells and indirectly through vascular injury. ${ }^{14}$ Direct parenchymal injury becomes evident at around 12 months, however, vascular injury usually presents late. Radiation may inhibit specific functions of the follicular epithelium, reduce the number of functional follicles, alter vascular permeability and induce immunological damage. However, the exact mechanism is unknown. ${ }^{15}$

In our study, $81.71 \%$ patients had a minimum follow-up of 6 months. Follow-up ranged from 6 months to 12 months with a median follow-up of 9 months. Kim et $\mathrm{al}^{7}$ had a median follow up of 25 months. The study by Einhorn et $\mathrm{al}^{16}$ had the longest follow-up of 10 years. The studies by Tell et al ${ }^{17}$ and Mercado et $\mathrm{al}^{18}$ each suggested that post radiation thyroid dysfunction presents between 1.4-1.8 months and hence, 1-2 years of follow up is required to adequately diagnose post radiation thyroid dysfunction. In our study, $85.37 \%$ were treated with chemoradiation, while $14.63 \%$ were treated with radiation alone. In the study conducted by Murthy et al ${ }^{19}$ 84.29\% patients were treated with chemoradiation and $15.71 \%$ were treated with radiation alone, which was similar to our study. In our study, $46(56.09 \%)$ patients received weekly Inj. Cisplatin $\left(40 \mathrm{mg} / \mathrm{m}^{2}\right), 15(18.29 \%)$ received three-weekly Inj. Cisplatin $\left(100 \mathrm{mg} / \mathrm{m}^{2}\right)$ and 9 patients received Inj. Carboplatin (AUC-2) on weekly basis in our study. In the study by Banipal et al $^{9} 41.5 \%$ received Inj. Cisplatin and 5-Fluorouracil and 5.6\% received Inj Cisplatin. In the study by Aich et al, ${ }^{20} 12.29 \%$ patients were given concurrent Cisplatin and 5-FU.

In our study, there was a decrease in the mean T3 levels from baseline to 6 months following which there was an increase from 9 months onwards. The reduction in mean T3 levels from baseline to 6 months was statistically significant. The change in T3 levels showed no significant correlation with mode of treatment, radiation dose or age group. In the study by Banipal et al, ${ }^{9} 60.3 \%$ had decrease in mean T3 levels and the change in the mean T3 levels according to age group, showed an uneven trend. There was no difference in mean T3 levels when correlated with mode of treatment. Chougule and Kochar ${ }^{21}$ showed that there was a decrease in mean T3 levels from baseline to mid-way of EBRT (30Gy). However, this decline was not statistically significant. The fall in mean T3 levels from baseline to 6 months after radiotherapy was found to be significant. In the study by Nishiyama et $a,^{22}$ there was a decrease in mean T3 in the hypothyroid group 12 months after irradiation however, it was not found to be statistically significant.

In our study, the change in serum mean T4 levels from baseline showed a decreasing trend until 9 months following which there was an increase in the T4 levels from 9 months to 12 months. The variation in $\mathrm{T} 4$ levels showed no correlation with age, mode of treatment or radiation dose. In the study by Vrabec et al,23 decrease in mean T4 levels were seen in $30.8 \%$ patients. In their study, the mean T4 levels were found to be lower in the RT- only group when compared to the chemoradiation group. The mean T4 levels were found to be higher in males when compared to females. The mean T4 levels were found to be higher in the group receiving $>60$ Gy. Chogule and Kochar ${ }^{21}$ found that mean T4 levels decreased from baseline to mid-way of EBRT (30Gy), however this was not statistically significant. The decrease from baseline was found to be significant only at 6 months post radiation.

We found that the incidence of hypothyroidism was $19.51 \%$ in our study. There was an increasing trend in the mean TSH levels, and the increase was found to be statistically significant at 3 months, 6 months and 12 months. There was a decrease in TSH levels in from 9 months to 12 months, likely due to administration of levo-thyroxine in our patients with hypothyroidism.

In the study by Nishiyama et al 22 TSH level did not change in the first 3 months but significantly increased from 3 months to 6 months. Koc and Capoglu ${ }^{24}$ observed the median time of 15 months for development of clinical hypothyroidism and 3 months for subclinical hypothyroidism. Our study did not find any statistically significant correlation between age and mean TSH levels. Mean TSH was found to statistically higher in males at baseline, while the difference at subsequent follow-up was not found to be statistically significant. There was no positive correlation when mean TSH levels were compared to primary site or use of chemotherapy along with radiation.

In our study, higher age was associated with a greater risk of hypothyroidism. $80 \%$ patients above 75 years of age developed hypothyroidism. According to the most researchers, age does not seem to be an important factor in hypothyroidism development. ${ }^{12,25,26}$ Only Tell et al ${ }^{17}$ found that increasing age was correlated with an increased risk of hypothyroidism. In a study by Srikantia et al, $2752.9 \%$ of the patients who developed clinical hypothyroidism were between the age groups of 51 to 60 years. In our study, the occurrence was definitely higher in patients older than 45 years of age. When patients developing hypothyroidism were assessed according to the radiation dose received, it was seen that In the patients receiving $<60 \mathrm{~Gy}, 33.33 \%$ patients developed hypothyroidism, in the group receiving $60 \mathrm{~Gy}$, $4.35 \%$ developed hypothyroidism and in the group receiving $>60 \mathrm{~Gy}, 24.53 \%$ developed hypothyroidism.

Dose of radiation required to produce hypothyroidism is not clearly defined. DeGroot ${ }^{28}$ and Hancock et al ${ }^{15}$ suggested that radiation doses in the range of 3000 to 8000 rads are required to produce hypothyroidism. In the study by Grande et $\mathrm{al}^{26}$ the incidence of hypothyroidism was $22.4 \%$ in patients receiving $<60$ Gy and $56 \%$ in those receiving $>60 \mathrm{~Gy}$. However, other studies like Alterio et $\mathrm{al}^{12}$ and Tell et al ${ }^{17}$ did not find any correlation between treatment dose and thyroid dysfunction. The incidence of hypothyroidism in the RT alone group was $33.33 \%$ and in the chemoradiation group it was $17.14 \%$. However, this difference was not found to be statistically significant.

The effect of chemotherapy on the thyroid gland, especially in conjunction with radiation is not clear. It is expected that chemotherapy, particularly concurrent chemotherapy will sensitize the thyroid gland to radiation 
and will increase the incidence of hypothyroidism. Hancock et $\mathrm{al}^{29}$ reported that chemotherapy was a significant risk factor for hypothyroidism in patients receiving radiation. On the other hand, Weissler and Berry ${ }^{8}$, Sinard et $\mathrm{al}^{30}$ and Mercado et al ${ }^{18}$ observed no increase in the incidence of hypothyroidism in patients with head and neck carcinoma who received chemotherapy as part of the treatment.

In our study, it was seen that the mean dose to the thyroid was higher in the hypothyroid group vs the euthyroid group (5004.69 \pm 161.91 vs $4643.44 \pm 825.05$ ), which was statistically significant ( $p$-value $<0.05$ ). The lowest mean dose at which thyroid dysfunction was seen, was at $4804 \mathrm{cGy}$. In the study by Ling et $\mathrm{al}^{31}$ the lowest mean dose at which thyroid abnormality occurred was noted to be at $29.8 \mathrm{~Gy}$. In the study by Kim et al 7 there was a positive correlation between higher mean dose to the thyroid and thyroid dysfunction. Bhandare et $\mathrm{al}^{10}{ }^{10}$ found a significant difference in the incidence of hypothyroidism between two different dose levels. When the mean thyroid gland dose was 45 Gy or more, the incidence of clinical hypothyroidism was $27 \%$ after 5 years and $39 \%$ after 10 years as compared to $21 \%$ and $21 \%$, respectively, in case of a mean thyroid gland dose less than $45 \mathrm{~Gy}$.

In our study, the dose volume parameters V10-V50 were not found to have any positive correlation with thyroid dysfunction. While V10, V20, V30, V50 were found to be higher in the hypothyroid group, these differences were not found to be statistically significant. In the study by Kim et al, ${ }^{7}$ V45 was the only variable that independently contributed to the prediction of hypothyroidism and suggested that V45 of $50 \%$ may be a possible dose-volumetric threshold of radiation-induced hypothyroidism. Similarly, Akgun et al ${ }^{6}$ also proposed that V30 may be a useful predictor of thyroid dysfunction in head and neck irradiation. Yoden et $\mathrm{al}^{32}$ reported that the correlation between the percentage of the volume receiving $10 \mathrm{~Gy}$ (V10), 20 Gy (V20) and $30 \mathrm{~Gy}$ (V30) is a possible predictor of hypothyroidism.

In our study, the mean thyroid volume in the hypothyroid group was $9.92 \pm 2.89$ compared to $12.75 \pm 4.78$ in the euthyroid group and hypothyroidism was more common in patients with smaller thyroid volumes. The study by Murthy et al $^{19}$ showed no correlation between thyroid volume and thyroid dysfunction. Jerezeck et $\mathrm{al}^{12}$, showed that smaller thyroid volume was associated with a higher incidence of thyroid toxicity.

All the cases of thyroid dysfunction comprised Subclinical hypothyroidism. There was an increase in TSH levels above the upper limit of the laboratory values. There was a decrease in T3 and T4 levels, however, these were found to be within the normal laboratory range.

\section{CONCLUSIONS}

Higher mean dose (above 48Gy) and smaller volume of thyroid gland had significant association with the development of hypothyroidism. TSH levels were significantly higher at baseline in males than in females but there was no significant correlation between gender and thyroid dysfunction. There was no association between thyroid dysfunction and the primary site or dose-volume parameters V10-V50. Thyroid dysfunction after head and neck cancer continues to be underestimated and under reported. The symptoms of thyroid dysfunction may go unnoticed under the cover of other associated co-morbidities and side effects of the treatment modalities. Regular testing of thyroid function in patients undergoing radiation or chemo radiation can help in diagnosing thyroid dysfunction at the subclinical stage, before it progresses to clinical hypothyroidism. Larger multi-institutional studies with longer follow-up are needed to study the morbidity associated with post radiation thyroid dysfunction further.

\section{REFERENCES}

[1] Jemal A, Bray F, Center MM, et al. Global cancer statistics. CA Cancer J Clin 2011;61(2):69-90.

[2] Takiar R, Nadayil D, Nandakumar A. Projections of number of cancer cases in India (2010-2020) by cancer groups. Asian Pac J Cancer Prev 2010;11(4):1045-9.

[3] Carvalho AL, Nishimoto IN, Califano JA, et al. Trends in incidence and prognosis for head and neck cancer in the United States: a site-specific analysis of the SEER database. Int J Cancer 2005;114(5):806-16.

[4] Pignon JP, Le Maitre A, Maillard E, et al. Meta-analysis of chemotherapy in head and neck cancer (MACH-NC): an update on 93 randomised trials and 17,346 patients. Radiotherapy and Oncology 2009;92 (1):4-14.

[5] Soberman N, Leonidas JC, Cherrick I, et al. Sonographic abnormalities of the thyroid gland in long term survivors of Hodgkin's disease. Pediatr Radiol 1991;21(4):250-3.

[6] Akgun Z, Atasoy BM, Ozen Z, et al. V30 as a predictor for radiation-induced hypothyroidism: a dosimetric analysis in patients who received radiotherapy to the neck. Radiation Oncology 2014;9:104.

[7] Kim MY, Yu T, Wu HG. Dose-volumetric parameters for predicting hypothyroidism after radiotherapy for head and neck cancer. Jpn J Clin Oncol 2014;44(4):331-7.

[8] Weissler MC, Berry BW. Thyroid stimulating hormone levels after radiotherapy and combined therapy for head and neck cancer. Head Neck 1991;13(5):420-3.

[9] Banipal R, Mahajan MK, Uppal B, et al. Thyroid disease as a sequelae following treatment of head and neck cancer. Indian J Cancer 2011;48(2):194-8.

[10] Bhandare N, Kennedy L, Malpaya RS, et al. Primary and central hypothyroidism after radiotherapy for head and neck tumours. Int $\mathrm{J}$ Radiat Oncol Biol Phys 2007;68(4):1131-9.

[11] Sachdev S, Refaat T, Bacchus ID, et al. Thyroid V50 highly predictive of hypothyroidism in head-and-neck cancer patients treated with Intensity-Modulated Radiotherapy (IMRT). Am J Clin Oncol 2017;40(4):4137.

[12] Alterio D, Jereczek-Fossa BA, Franchi B, et al. Thyroid disorders in patients treated with radiotherapy for head-and-neck cancer: a retrospective analysis of seventy-three patients. Int J Radiat Oncol Biol Phys 2007;67(1):144-50.

[13] Boomsma MJ, Bijl HP, Langendijk JA. Radiation-induced hypothyroidism in head and neck cancer patients: a systematic review. Radiat Oncol 2011;99(1):1-5. 
[14] Rubin P, Casarett GW. Clinical radiation pathology as applied to curative radiotherapy. Cancer 1968;22(4):767-78.

[15] Hancock SL, McDougall IR, Constine LS. Thyroid abnormalities after therapeutic external radiation. Int J Radiat Oncol Biol Phys 1995;31(5):1165-70.

[16] Einhorn J, Wilkholm G. Hypothyroidism after external irradiation to the thyroid region. Radiology 1967;88(2):326-8.

[17] Tell R, Lundell G, Nilsson B, et al. Long-term incidence of hypothyroidism after radiotherapy in patients with head-and-neck cancer. Int J Radiat Oncol Biol Phys 2004;60(2):395-400.

[18] Mercado G, Adelstein DJ, Saxton JP, et al. Hypothyroidism: a frequent event after radiotherapy and after radiotherapy with chemotherapy for patients with head and neck carcinoma. Cancer 2001;92(11):2892-7.

[19] Murthy V, Narang K, Ghosh-Laskar S, et al. Hypothyroidism after 3-dimensional conformal radiotherapy and intensity-modulated radiotherapy for head and neck cancers: prospective data from 2 randomized controlled trials. Head \& Neck 2014;36(11):1573-80.

[20] Aich RK, Deb AR, Pal S, et al. Iatrogenic hypothyroidism: a consequence of external beam radiotherapy to the head \& neck malignancies. J Cancer Res Ther 2005;1(3):142-6.

[21] Chougule A, Kochar B. Thyroid dysfunction following therapeutic external radiation to head and neck cancer. Asian Pacific J Cancer Prev 2011;12(2):443-5.

[22] Nishiyama K, Kozuka T, Higashihara T, et al. Acute radiation thyroiditis. Int $\mathrm{J}$ Radiat Oncol Biol Phys 1996;36(5):1221-4.

[23] Vrabec DP, Heffron TJ. Hypothyroidism following treatment for head and neck cancer. Ann Otol Rhinol Laryngol 1981;90(5 Pt 1):449-53.
[24] Koc M, Capoglu I. Thyroid dysfunction in patients treated with radiotherapy for neck. Am J Clin Oncol 2009;32(2):150-3.

[25] Posner MR, Ervin TJ, Miller D, et al. Incidence of hypothyroidism following multimodality treatment for advanced squamous cell cancer of the head and neck. Laryngoscope 1984;94(4):451-4.

[26] Grande C. Hypothyroidism following radiotherapy for head and neck cancer: multivariate analysis of risk factors. Radiother Oncol 1992;25(1):31-6.

[27] Srikantia N, Rishi KS, Janaki MG, et al. How common is hypothyroidism after external radiotherapy to neck in head and neck cancer patients? Indian Journal of Medical and Paediatric Oncology: Official Journal of Indian Society of Medical \& Paediatric Oncology 2011;32(3):143-8.

[28] De Groot LJ. Radiation and thyroid disease. Bailliere Clin Endocrinol Metabol 1988;2(3):777-91.

[29] Turner SL, Tiver KW, Boyages SC. Thyroid dysfunction following radiotherapy for head and neck cancer. Int J Radiat Oncol Biol Phys 1995;31(2):279-83.

[30] Sinard RJ, Tobin EJ, Mazzaferri EL, et al. Hypothyroidism after treatment for nonthyroid head and neck cancer. Arch Otolaryngol Head Neck Surg 2000;126(5):652-7.

[31] Ling S, Bhatt AD, Brown NV, et al. Correlative study of dose to thyroid and incidence of subsequent dysfunction after head and neck radiation. Head \& Neck 2017;39(3):548-54.

[32] Yoden E, Soejima T, Maruta T, et al. Hypothyroidism after radiotherapy to the neck. Nippon Igaku Hoshasen Gakkai Zasshi 2004;64(3):146-50. 